\title{
Axial convection of two immiscible fluids in a vertical tube
}

Cite as: AIP Conference Proceedings 2164, 050001 (2019); https://doi.org/10.1063/1.5130799

Published Online: 24 October 2019

V. K. Andreev, and E. P. Magdenko

\section{ARTICLES YOU MAY BE INTERESTED IN}

Solution of the problems of nonhomogeneous incompressible fluid dynamics by the CABARET method

AIP Conference Proceedings 2164, 120007 (2019); https://doi.org/10.1063/1.5130867

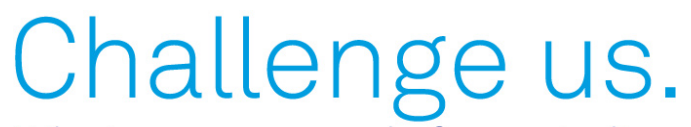

What are your needs for periodic

signal detection?

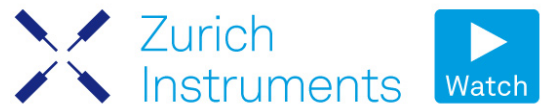

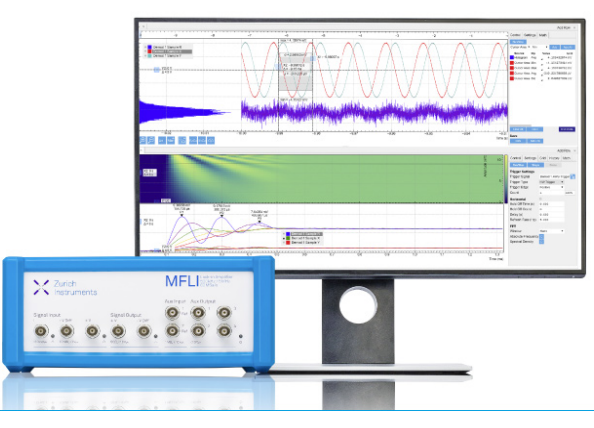

2164, 050001 


\title{
Axial Convection of Two Immiscible Fluids in a Vertical Tube
}

\author{
V. K. Andreev ${ }^{1,2, \text { a) }}$ and E. P. Magdenko ${ }^{1,2, \text { b) }}$ \\ ${ }^{1)}$ Institute of Computational Modeling of the Siberian Branch of the Russian Academy of Sciences, 50/44 Akademgorodok, \\ 660036 Krasnoyarsk, Russia \\ ${ }^{2)}$ Institute of Mathematics and Fundamental Informatics of the Siberian Federal University, 79 Svobodny, 660041 Krasnoyarsk, \\ Russia \\ a)Electronic mail: andr@icm.krasn.ru \\ b)Corresponding author: magdenko_evgeniy@icm.kras.ru
}

\begin{abstract}
The conjugate initial-boundary inverse problem of unidirectional motion of two immiscible liquids in a vertical tube with cylindrical solid wall is investigated. The fluids form cylindrical interface. The gravity acceleration acts on the fluids. The stationary solution of problem is found. It is shown that if the temperature on the solid tube wall uniformly tends to a stationary value with increasing time, then the solutions of the non-stationary problem with increasing time tend to a steady state.
\end{abstract}

\section{INTRODUCTION}

The problems about the convection formation in a liquid are great practical interest. It is known that motion occurs in a non-uniformly heated fluid, and it often happens in two or more liquid media that interact along interfaces. Currently, interest in the models of multiphase flows, taking into account the differences in physical and chemical factors, arises in the designing of cooling systems and power plants, the growth of crystals and films, and the aerospace industry [1]. The study of such problems is associated with great mathematical difficulties: the nonlinearity of equations and boundary conditions on the interfaces, the unknown fields of solutions definition. In an experimental study, the difficulties are mainly caused in the conditions reconstruction in which there is a specific phenomenon. Therefore, the mathematical modeling methods are now considered to be an important alternative for studying an extremely wide range of convective flow problems. It is also important to choose a correct mathematical model for studying a particular phenomenon. In this way, it is possible to determine in advance those problem parameters where particular processes prevail. For instance, the Oberbeck-Boussinesq model, which is often used to study natural convection problems, becomes inapplicable under microgravity conditions or in microscopic-scale problems. But these equations admit a solutions where the temperature and pressure are linear functions of one Cartesian coordinate, while all three components of velocity are independent of this coordinate. These solutions are interpreted as flows in infinitely-long tubes with an arbitrary cross section. They generalize the known Birikh's solution of a plane problem of convection in a horizontal band [2].

The liquid that undergoes nonlinear Marangoni instability in a circular cylinder is considered [3]. In the paper [4] a cylinder of finite dimensions in the gravity field was considered. The free boundary was also assumed to be undeformable. The authors considered two cases, namely, that in which the lateral wall of the vessel was adiabatically thermally-insulated and that in which temperature disturbances vanish on this wall. In paper [5] the authors considered the linear stability of an incompressible axisymmetric flow of a thermocapillary fluid in a cylinder of finite size, which is heated on top by a heat flux having a parabolic radial profile. For the Oberbeck-Boussinesq model, the layer stability with a linear dependence of the Rayleigh number on the Marangoni number was studied, for example, in the paper [6]

In this paper the problem about unidirectional motion of two immiscible heat-conducting fluids with a cylindrical interface is considered. This problem was first formulated by V. V. Pukhnachev in 2012 [7]. The author assumed that one of the pressure gradients is a known quantity, and the second was determined from the dynamic condition specified on the interface. In this paper, the pressure gradients are the sought quantities together with the functions of speed and temperature. Thus the problem is the inverse. Therefore, for complete definiteness of the problem, either the flow rate of the first fluid or the total fluids flow rate is given.

\section{PROBLEM STATEMENT}

Consider the unidirectional convective motion of two immiscible liquids in a vertical tube of radius $R_{2}$ with cylindrical solid wall. Fluids form a cylindrical interface Figure 1. The liquid $l$ moves in the cylinder $0 \leqslant r \leqslant R_{1}$ and the liquid 2 moves in the annular layer $R_{1} \leqslant r \leqslant R_{2}$. The gravity acceleration $\mathbf{g}=(0,0,-g)$ acts on the fluids.

Application of Mathematics in Technical and Natural Sciences

AIP Conf. Proc. 2164, 050001-1-050001-9; https://doi.org/10.1063/1.5130799

Published by AIP Publishing. 978-0-7354-1909-4/\$30.00 


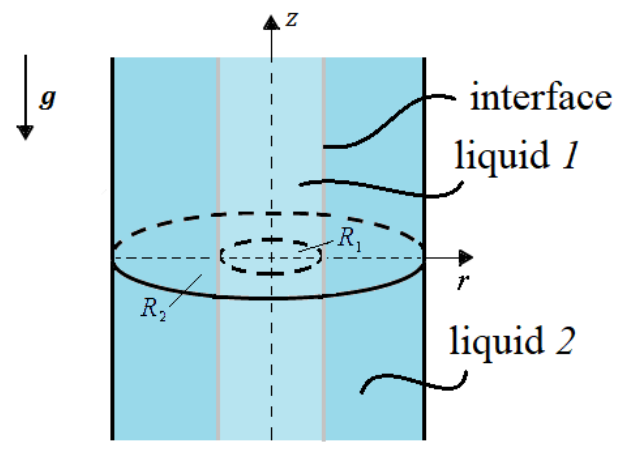

FIGURE 1. Scheme of flow field

The solution of the Oberbeck-Boussinesq equations is sought in the form

$$
\begin{gathered}
\mathbf{u}_{j}=\left(0,0, w_{j}(r, t)\right), \quad \theta_{j}=-A_{j}(r, t) z+T_{j}(r, t), \\
p_{j}=-B_{j}(r, t) z+q_{j}(r, t), \quad j=1,2 .
\end{gathered}
$$

where $j$ denotes number of liquids, $\mathbf{u}_{j}$ is velocity vector, $\theta_{j}$ is temperature, $p_{j}$ is pressure. Substituting the form of solution (1) into the Oberbeck-Boussinesq system leads to equations for the functions $w_{j}, A_{j}, T_{j}$ :

$$
\begin{aligned}
w_{j t} & =v_{j}\left(w_{j r r}+\frac{1}{r} w_{j r}\right)+g \beta_{j} \int_{R_{1}}^{r} s A_{j}(s, t) d s-\frac{1}{\rho_{j}} F_{j}(t), \\
A_{j t} & =\chi_{j}\left(A_{j r r}+\frac{1}{r} A_{j r}\right) \\
T_{j t} & =\chi_{j}\left(T_{j r r}+\frac{1}{r} T_{j r}\right)+A_{j} w_{j}
\end{aligned}
$$

where $t>0$ and $j=1$ on interval $0<r<R_{1}$ and $j=2$ on interval $R_{1}<r<R_{2} ; \rho_{j}, v_{j}, \chi_{j}$ are positive constant density, kinematic viscosity and thermal diffusivity, respectively, $\beta_{j}$ is fluid thermal expansion coefficient.

Functions $B_{j}(r, t)$ and $q_{j}(r, t)$ can be found using quadratures based on known functions $A_{j}(r, t)$ and $T_{j}(r, t)$

$$
B_{j}=\rho_{j} g \beta_{j} \int_{R_{1}}^{r} s A_{j}(s, t) d s-F_{j}(t), \quad q_{j}=\rho_{j} g \beta_{j} \int_{R_{1}}^{r} s T_{j}(s, t) d s+C_{j}(t) .
$$

The initial conditions are posed for unknown functions $w_{j}(r, t), A_{j}(r, t), T_{j}(r, t)$

$$
\begin{aligned}
& w_{1}(r, 0)=w_{01}(r), \quad A_{1}(r, 0)=A_{01}(r), \quad T_{1}(r, 0)=T_{01}(r) \quad \text { at } \quad 0 \leqslant r \leqslant R_{1} \text {; } \\
& w_{2}(r, 0)=w_{02}(r), \quad A_{2}(r, 0)=A_{02}(r), \quad T_{2}(r, 0)=T_{02}(r) \quad \text { at } \quad R_{1} \leqslant r \leqslant R_{2} \text {. }
\end{aligned}
$$

Also the conditions are posed on the lateral surface of the cylinder

$$
w_{2}\left(R_{2}, t\right)=0, \quad A_{2}\left(R_{2}, t\right)=\gamma(t), \quad T_{2}\left(R_{2}, t\right)=\delta(t) \quad \text { at } \quad t>0
$$

with given functions $\gamma(t), \delta(t)$.

On the axis of the inner cylinder the functions $w_{1}(r, t), A_{1}(r, t)$ and $T_{1}(r, t)$ should be limited

$$
\left|w_{1}(0, t)\right|<\infty, \quad\left|A_{1}(0, t)\right|<\infty, \quad\left|T_{1}(0, t)\right|<\infty .
$$

The following conditions are fulfilled on the interface

$$
\begin{gathered}
w_{1}\left(R_{1}, t\right)=w_{2}\left(R_{1}, t\right), \quad A_{1}\left(R_{1}, t\right)=A_{2}\left(R_{1}, t\right), \quad T_{1}\left(R_{1}, t\right)=T_{2}\left(R_{1}, t\right), \\
k_{1} A_{1 r}\left(R_{1}, t\right)=k_{2} A_{2 r}\left(R_{1}, t\right), \quad k_{1} T_{1 r}\left(R_{1}, t\right)=k_{2} T_{2 r}\left(R_{1}, t\right), \\
\rho_{1} v_{1} w_{1 r}-\rho_{2} v_{2} w_{2 r}=æ A_{1}\left(R_{1}, t\right) .
\end{gathered}
$$


Here the last condition is the balance condition of shear stresses and thermo-capillary forces. The parameter $æ>0$ is coefficient of linear dependence of surface tension on temperature $\left(\sigma\left(\theta_{1}\right)=\sigma^{0}-\mathfrak{x}\left(\theta_{1}\left(R_{1}, t\right)-\theta_{0}\right)\right.$ with constant $\left.\sigma^{0}>0, \theta_{0}>0\right)$. In connection with this fact and in that the dynamic conditions $\left(p_{1}\left(R_{1}, z, t\right)-p_{2}\left(R_{1}, z, t\right)=\sigma\left(\theta_{1}\right) / R_{1}\right)$ are fulfilled on interface, we obtain

$$
\begin{aligned}
& F_{1}(t)=F_{2}(t)+\frac{æ A_{1}\left(R_{1}, t\right)}{R_{1}}, \\
& K_{1}(t)=K_{2}(t)+\frac{\sigma^{0}-æ\left(T_{1}\left(R_{1}, t\right)-\theta_{0}\right)}{R_{1}} .
\end{aligned}
$$

For smooth solutions, the matching conditions must be fulfilled

$$
\begin{gathered}
w_{01}\left(R_{1}\right)=w_{02}\left(R_{1}\right), \quad w_{02}\left(R_{2}\right)=0, \\
A_{01}\left(R_{1}\right)=A_{02}\left(R_{1}\right), \quad T_{01}\left(R_{1}\right)=T_{02}\left(R_{1}\right), \\
k_{1} \frac{\partial A_{01}\left(R_{1}\right)}{\partial r}=k_{2} \frac{\partial A_{02}\left(R_{1}\right)}{\partial r}, \quad k_{1} \frac{\partial T_{01}\left(R_{1}\right)}{\partial r}=k_{2} \frac{\partial T_{02}\left(R_{1}\right)}{\partial r}, \\
\rho_{1} v_{1} \frac{\partial w_{01}\left(R_{1}\right)}{\partial r}-\rho_{2} v_{2} \frac{\partial w_{02}\left(R_{1}\right)}{\partial r}=æ A_{01}\left(R_{1}\right) .
\end{gathered}
$$

If the function $F_{2}(t)$ (or $F_{1}(t)$ ) is pose then the problem statement is completed. The equality (11) means that the pressure in one of the liquids is up to an arbitrary time function. This problem was first formulated by Professor V. V. Pukhnachev, see [7, p. 371-373].

In this paper, we are interested in the case when $F_{1}(t)$ is sought together with the functions $w_{j}(r, t), A_{j}(r, t), T_{j}(r, t)$. To do this, we pose the flow rate of the first fluid.

$$
\int_{0}^{R_{1}} r w_{1}(r, t) d r=\frac{Q(t)}{2 \pi} .
$$

In some applications, the total volume flow rate of liquids is known

$$
\int_{0}^{R_{1}} r w_{1}(r, t) d r+\int_{R_{1}}^{R_{2}} r w_{2}(r, t) d r=\frac{Q(t)}{2 \pi} .
$$

Remark 1. The obtained problems are conjugate and inverse because the pressure gradients $\left(F_{j}(t)\right)$ are also sought.

Remark 2. The initial-boundary value problem for functions $w_{j}(r, t), A_{j}(r, t), F_{j}(t)$ is closed subsystem. The problem for the function $T_{j}(r, t)$ is separated. This functions do not affect the velocity behavior.

\section{STEADY STATE}

Suppose that $\gamma(t)=\gamma_{0}=$ const, $\delta(t)=\delta_{0}=$ const, $K_{j}(t)=$ const, $Q(t)=Q_{0}=$ const. Then we can look for a stationary solutions $w_{j}^{s}(r), A_{j}^{s}(r), F_{j}^{s}=$ const. To begin with, dimensionless variables are introduced. The scales of length, time, speed, temperature, pressure gradient are as follows:

$$
\begin{gathered}
R_{1}, \frac{R_{1}^{2}}{\chi_{1}}, \frac{\chi_{1}}{R_{1}}, \gamma_{0} R_{1}, \frac{\rho_{1} v_{1} \chi_{1}}{R_{1}^{3}} \quad \text { at } 0 \leqslant r \leqslant R_{1} ; \\
R_{2}-R_{1}, \frac{R_{1}^{2}}{\chi_{1}}, \frac{\chi_{2}}{R_{2}-R_{1}}, \gamma_{0}\left(R_{2}-R_{1}\right), \frac{\rho_{2} v_{2} \chi_{2}}{\left(R_{2}-R_{1}\right)^{3}} \quad \text { at } \quad R_{1} \leqslant r \leqslant R_{2} .
\end{gathered}
$$

For the case when the temperature on the side surface of the cylinder and the flow rate condition (14) are posed, we obtain, taking into account conditions (9)

$$
A_{1}^{s}=A_{2}^{s}=1
$$




$$
\begin{aligned}
& W_{1}^{s}=-\frac{1}{32} \operatorname{Ra}_{1}\left(\frac{\xi^{4}}{4}-\xi^{2}\right)+\frac{1}{4} F_{1}^{s} \xi^{2}+C_{1}, \quad 0 \leqslant \xi \leqslant 1, \\
& W_{2}^{s}=-\frac{1}{32} \operatorname{Ra}_{2}\left(\frac{\eta^{4}}{4}-\frac{4 \varepsilon^{2}}{(1-\varepsilon)^{2}} \eta^{2}\right)+\frac{1}{4} F_{2}^{s} \eta^{2}+C_{2} \ln \left(\frac{1-\varepsilon}{\varepsilon} \eta\right)+C_{3}, \quad \frac{\varepsilon}{1-\varepsilon} \leqslant \eta \leqslant \frac{1}{1-\varepsilon},
\end{aligned}
$$

where $\mathrm{Ra}_{1}=g \beta_{1} \gamma_{0} R_{1}^{4} / v_{1} \chi_{1}$ and $\mathrm{Ra}_{2}=g \beta_{2} \gamma_{0}\left(R_{2}-R_{1}\right)^{4} / v_{2} \chi_{2}$ are Rayleigh numbers, $\varepsilon=R_{1} / R_{2}$. The constants $C_{m}$, $m=1, . .3$ are determined from conditions (8), (10). Taking into account conditions of the flow rate of the first fluid (14) and condition (11) the pressure gradients are determined as

$$
\begin{aligned}
& F_{1}^{s}=\frac{1}{\rho v \chi} \frac{\varepsilon^{3}}{(1-\varepsilon)^{3}} F_{2}^{s}+\mathrm{Ma}, \\
& F_{2}^{s}=\frac{8 \rho v \chi(1-\varepsilon)^{3}}{\varepsilon\left(2 \rho v\left(\varepsilon^{2}-1\right)-\varepsilon^{2}\right)}\left(\frac{\bar{Q}}{\pi}+\frac{1}{8} \mathrm{Ma}+\frac{1}{24} \mathrm{Ra}_{1}-\frac{1}{32} \frac{\varepsilon\left(3 \varepsilon^{4}-4 \varepsilon^{2}+1\right)}{\chi(1-\varepsilon)^{5}} \mathrm{Ra}_{2}-\frac{\varepsilon \ln (\varepsilon)}{\chi(1-\varepsilon)} C_{2}\right) .
\end{aligned}
$$

Here $\rho=\rho_{1} / \rho_{2}, v=v_{1} / v_{2}, \beta=\beta_{1} / \beta_{2}, \chi=\chi_{1} / \chi_{2}, \mathrm{Ma}=\mathfrak{x} \gamma_{0} R_{1}^{2} / \rho_{1} v_{1} \chi_{1}$ is Marangoni number. If the total volume fluids flow rate (15) is posed then $F_{2}^{s}=Y / X$ where

$$
\begin{aligned}
& X=\frac{2 \rho v\left(\varepsilon^{3}-\varepsilon(\chi+1)-\chi\right)-\varepsilon^{3}}{\rho v \chi(1-\varepsilon)^{3}}+\frac{1-\varepsilon^{4}}{(1-\varepsilon)^{4}} \\
& Y=\frac{8 \bar{Q}}{\pi}+\frac{1}{3} \operatorname{Ra}_{1}+\operatorname{Ma}+8\left(\frac{\varepsilon^{2}(2 \ln (\varepsilon)-1)+1}{2(1-\varepsilon)^{2}}-\frac{\varepsilon \ln (\varepsilon)}{\chi(1-\varepsilon)}\right) C_{2} \\
& \quad+\frac{1}{12} \frac{\chi\left(5 \varepsilon^{4}+10 \varepsilon^{3}+3 \varepsilon^{2}-4 \varepsilon-2\right)+3 \varepsilon(\varepsilon+1)\left(3 \varepsilon^{2}-1\right)}{\chi(1-\varepsilon)^{4}} \mathrm{Ra}_{2} .
\end{aligned}
$$

Further for calculation the \{"glycerin", "silicone oill"\} system are chosen. This fluids have the following parameters

$$
\begin{gathered}
\rho=\{1.25,0.935\} \mathrm{kg} / \mathrm{cm}^{3}, \quad v=\{1.44,0.1\} \mathrm{cm}^{2} / \mathrm{c}, \\
\chi=\{0.0009,0.00096\} \mathrm{cm}^{2} / \mathrm{c}, \quad \beta=\{0.00061,0.00108\} \mathrm{K}^{-1}, \\
k=\{28000,13400\} \mathrm{W} /(\mathrm{cm} \cdot \mathrm{K}), \quad \mathfrak{x}=0.0598 \mathrm{~N} /(\mathrm{cm} \cdot \mathrm{K})
\end{gathered}
$$

Also the following parameters are posed

$$
\varepsilon=0.9, \quad R_{2}=0.1 \mathrm{~cm}, \quad g=981 \mathrm{~cm} / \mathrm{c}^{2} .
$$

Let be the total volume fluids flow rate (15) is posed.

We consider the dependence of the stationary velocity profile on the radial coordinate for different values of problem parameters. On the below figures taking into account the replacement of $\eta=\xi-(1-2 \varepsilon) /(1-\varepsilon)$ the velocity profiles $\chi(1-\varepsilon) W_{1}^{s}(\xi) / \varepsilon$ and $W_{2}^{s}(\eta)$ are shown respectively on intervals $0<\xi<1$ and $1<\xi<2$. It is established that with increasing parameter value $Q$ the velocity value grows (Figure 2). At increasing values of the temperature gradient $\gamma_{0}$ given on the side surface of the cylinder at the beginning the velocity value decreases and then it grows (Figure 3 ). Also it is established that the changes of the gravity acceleration does not almost influence on the velocity value.

It is worth noting that if we pose condition of first fluid flow rate (14) then we obtain the similar figures but the velocity value will be higher.

\section{SOLUTION OF THE PROBLEM BY THE LAPLACE TRANSFORMATION}

For finding the solution of the conjugate initial-boundary problem (2), (3), (6)-(11), (13)-(15) the separation method of variables is not applicable. We use the Laplace transformation [8]. Let

$$
\hat{u}(r, s)=\int_{0}^{\infty} u(r, t) e^{-s t} d t
$$




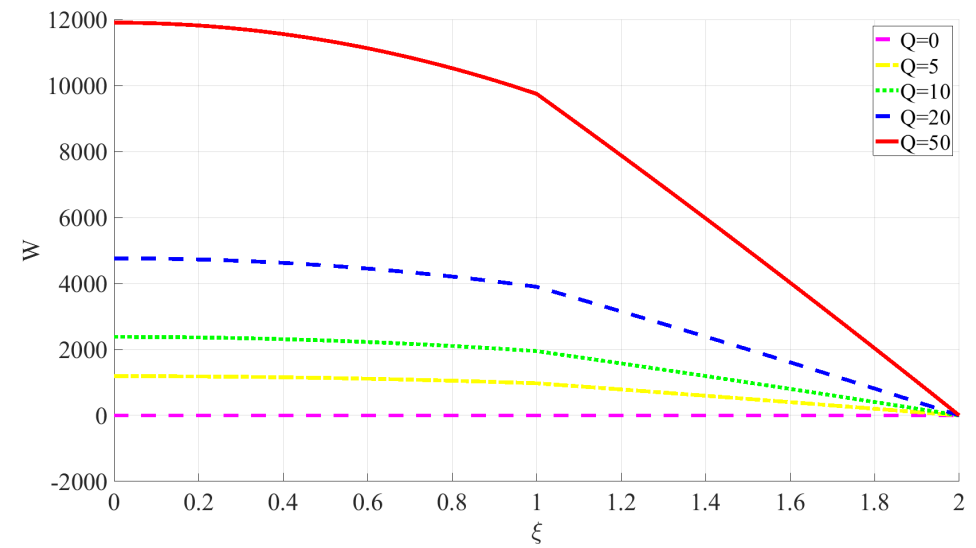

FIGURE 2. The dependence of the stationary velocity profile on the radial coordinate for different values of the volume liquids flow rate

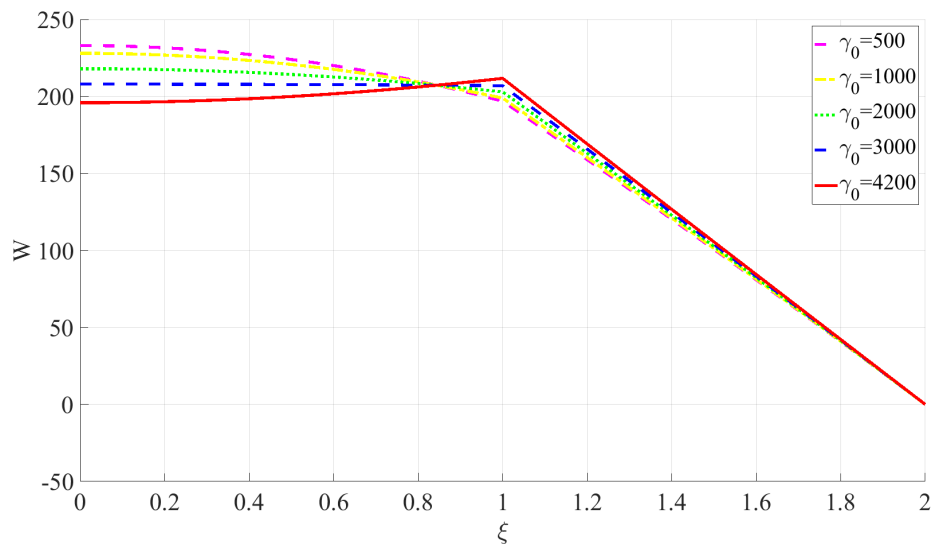

FIGURE 3. The dependence of the stationary velocity profile on the radial coordinate for different values of the temperature gradient given on the side surface of the cylinder.

be Laplace transformation of function $u(r, t)$. Then the problem mentioned above is reduced to the boundary value problem for the ODE system

$$
\begin{gathered}
\hat{A}_{1 \xi \xi}+\frac{1}{\xi} \hat{A}_{1 \xi}-s \hat{A}_{1}=-A_{01}(\xi), \\
\hat{A}_{2 \eta \eta}+\frac{1}{\eta} \hat{A}_{2 \eta}-\frac{\chi(1-\varepsilon)^{2}}{\varepsilon^{2}} s \hat{A}_{2}=-\frac{\chi(1-\varepsilon)^{2}}{\varepsilon^{2}} A_{02}(\eta), \\
\hat{W}_{1 \xi \xi}+\frac{1}{\xi} \hat{W}_{1 \xi}-\frac{s}{\operatorname{Pr}_{1}} \hat{W}_{1}=-\frac{1}{\operatorname{Pr}_{1}} W_{01}-\operatorname{Ra}_{1} \int_{1}^{z} z \hat{A}_{1} d z+\hat{F}_{1}(s), \\
\hat{W}_{2 \eta \eta}+\frac{1}{\eta} \hat{W}_{1 \eta}-\frac{s \chi}{\operatorname{Pr}_{2}} \frac{(1-\varepsilon)^{2}}{\varepsilon^{2}} \hat{W}_{2}=-\frac{\chi}{\operatorname{Pr}_{2}} \frac{(1-\varepsilon)^{2}}{\varepsilon^{2}} W_{02}-\operatorname{Ra}_{2} \int_{\varepsilon /(1-\varepsilon)}^{\eta} z \hat{A}_{2} d z+\hat{F}_{2}(s),
\end{gathered}
$$

where $0<\xi<1$ and $\varepsilon /(1-\varepsilon)<\eta<1 /(1-\varepsilon) ; \operatorname{Pr}_{j}=v_{j} / \chi_{j}$ are Prandtl numbers. 
The functions $\hat{W}_{1}(\xi, s), \hat{A}_{1}(\xi, s)$ are limited at $\xi=0$. Further

$$
\begin{aligned}
& \hat{W}_{2}\left(\frac{1}{1-\varepsilon}, s\right)=0, \quad \hat{A}_{2}\left(\frac{1}{1-\varepsilon}, s\right)=\hat{\gamma}(s), \\
& \hat{W}_{1}(1, s)=\frac{1}{\chi} \frac{\varepsilon}{1-\varepsilon} \hat{W}_{2}\left(\frac{\varepsilon}{1-\varepsilon}, s\right), \quad \hat{A}_{1}(1, s)=\hat{A}_{2}\left(\frac{\varepsilon}{1-\varepsilon}, s\right) \text {, } \\
& \hat{A}_{1 \xi}(1, s)=\frac{1}{k} \frac{\varepsilon}{1-\varepsilon} \hat{A}_{2 \eta}\left(\frac{\varepsilon}{1-\varepsilon}, s\right) \text {, }
\end{aligned}
$$

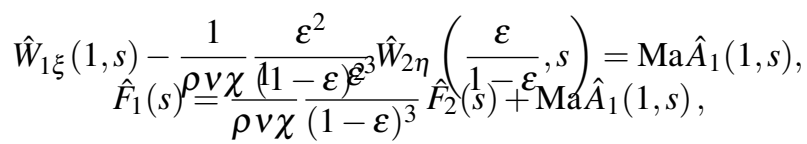

$$
\begin{aligned}
& \int_{0}^{1} \xi \hat{W}_{1}(\xi, s) d \xi=\frac{\hat{\bar{Q}}(s)}{2 \pi} \quad \text { or } \quad \int_{0}^{1} \xi \hat{W}_{1}(\xi, s) d \xi+\frac{1-\varepsilon}{\chi \varepsilon} \int_{\varepsilon /(1-\varepsilon)}^{1 /(1-\varepsilon)} \eta \hat{W}_{2}(\eta, s) d \eta=\frac{\hat{\bar{Q}}(s)}{2 \pi} .
\end{aligned}
$$

Remark 3. If one of the functions $\gamma(t), Q(t)$ is defined only on a finite time interval $\left[0, t_{1}\right]$ then then to apply the Laplace transform it can be continued with zero $t>t_{1}$ [8].

The boundary problem (22) for the functions $\hat{A}_{j}$ has a solution

$$
\begin{aligned}
& \hat{A}_{1}(\xi, s)=C_{1} I_{0}\left(a_{1} \xi\right)+\int_{1}^{\xi} x\left[K_{0}\left(a_{1} \xi\right) I_{0}\left(a_{1} x\right)-I_{0}\left(a_{1} \xi\right) K_{0}\left(a_{1} x\right)\right] A_{01} d x \\
& \hat{A}_{2}(\eta, s)=C_{2} I_{0}\left((1-\varepsilon) d_{1} \eta\right)+C_{3} K_{0}\left((1-\varepsilon) d_{1} \eta\right) \\
& \quad+\int_{\varepsilon /(1-\varepsilon)}^{\eta} x\left[K_{0}\left((1-\varepsilon) d_{1} \eta\right) I_{0}\left((1-\varepsilon) d_{1} x\right)-I_{0}\left((1-\varepsilon) d_{1} \eta\right) K_{0}\left((1-\varepsilon) d_{1} x\right)\right] A_{02} d x .
\end{aligned}
$$

Here $I_{0}(x), K_{0}(x)$ are modified Bessel functions of the first and second kind and

$$
a_{1}=\sqrt{s}, \quad d_{1}=\frac{\sqrt{s \chi}}{\varepsilon} .
$$

The constants $C_{1}, C_{2}, C_{3}$ are determined from boundary conditions (24).

Assuming that in the right sides of the equations (23) the functions $\hat{A}_{j}$ are already known the boundary problem (23) for the functions $\hat{W}_{j}$ has a solution

$$
\begin{aligned}
\hat{W}_{1}(\xi, s) & =D_{1} I_{0}\left(a_{2} \xi\right)+\int_{1}^{\xi} x\left[K_{0}\left(a_{2} \xi\right) I_{0}\left(a_{2} x\right)-I_{0}\left(a_{2} \xi\right) K_{0}\left(a_{2} x\right)\right] h_{1}(x, s) d x-\frac{1}{a_{2}^{2}} \hat{F}_{1}, \\
\hat{W}_{2}(\eta, s) & =D_{2} I_{0}\left((1-\varepsilon) d_{2} \eta\right)+D_{3} K_{0}\left((1-\varepsilon) d_{2} \eta\right) \\
& +\int_{\varepsilon /(1-\varepsilon)}^{\eta} x\left[K_{0}\left((1-\varepsilon) d_{2} \eta\right) I_{0}\left((1-\varepsilon) d_{2} x\right)-I_{0}\left((1-\varepsilon) d_{2} \eta\right) K_{0}\left((1-\varepsilon) d_{2} x\right)\right] h_{2}(x, s) d x-\frac{1}{(1-\varepsilon)^{2} d_{2}^{2}} \hat{F}_{2},
\end{aligned}
$$

where

$$
\begin{aligned}
h_{1}(\xi, s) & =\operatorname{Ra}_{1} \int_{1}^{\xi} x \hat{A}_{1}(x, s) d z+\frac{1}{\operatorname{Pr}_{1}} W_{01}, \\
h_{2}(\eta, s) & =\operatorname{Ra}_{2} \int_{\varepsilon /(1-\varepsilon)}^{\eta} x \hat{A}_{2}(x, s) d z+\frac{1}{\operatorname{Pr}_{2}} \frac{(1-\varepsilon)^{2}}{\varepsilon^{2}} W_{02}, \\
a_{2} & =\sqrt{\frac{s}{\operatorname{Pr}_{1}}}, \quad d_{2}=\frac{1}{\varepsilon} \sqrt{\frac{s \chi}{\operatorname{Pr}_{2}}} .
\end{aligned}
$$


The constants $D_{1}, D_{2}, D_{3}$ are determined from boundary conditions (24).

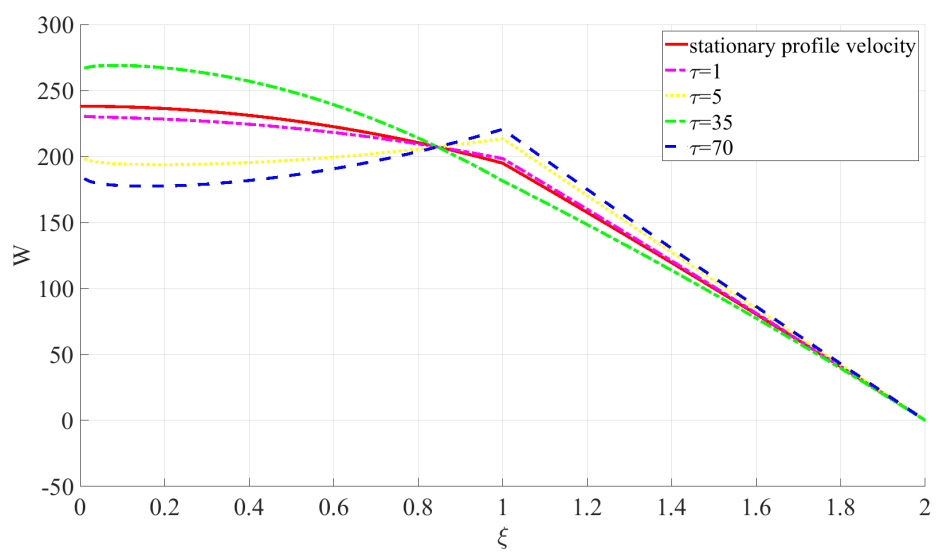

FIGURE 4. The dependence of the velocity profile on the radial coordinate at different values of the parameter $\tau$ when $\gamma(\tau)=1+10000 \sin (0.1 \tau)$

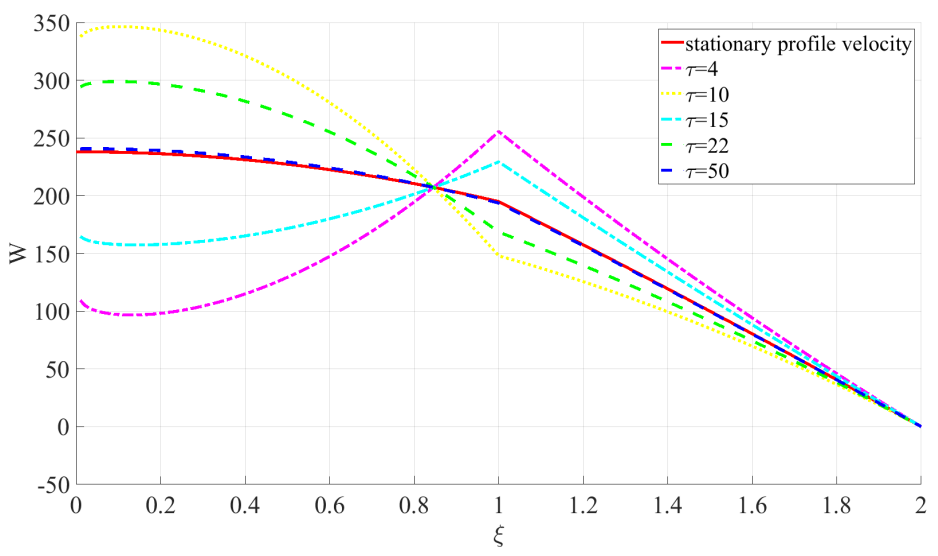

FIGURE 5. The dependence of the velocity profile on the radial coordinate at different values of the parameter $\tau$ when $\gamma(\tau)=1-20000 \exp (-0.05 \tau) \sin (0.5 \tau)$

Lemma. If finite limits for functions $\gamma(\tau)$ and $Q(\tau)$ at $\tau \rightarrow \infty$ exist in that

$$
\lim _{\tau \rightarrow \infty} \gamma(\tau)=\gamma_{0}, \quad \lim _{\tau \rightarrow \infty} Q(\tau)=Q_{0} .
$$

and the Laplace transformation is applicable to the derivatives of these functions then

$$
\lim _{s \rightarrow 0} s \hat{\gamma}(s)=\lim _{\tau \rightarrow \infty} \gamma(\tau)=\gamma_{0}, \quad \lim _{s \rightarrow 0} s \hat{Q}(s)=\lim _{\tau \rightarrow \infty} Q(\tau)=Q_{0},
$$

Theorem. If conditions (30) and (31) are fulfilled then the solution constructed in the Laplace images (27), (28) tends to a steady regime (16), (17) with increasing time.

Again we consider the liquids system \{ "glycerin", "silicone oil"\}. If the parameter $Q=1$ and the temperature gradient $(\gamma(\tau))$ given on the side surface of the cylinder is not fulfilled the lemma condition then the velocity profile does not tend to a steady regime (Figure 4). If the function $\gamma(\tau)$ has the following form $\gamma(\tau)=1-20000 \exp (-0.05 \tau) \sin (0.5 \tau)$ then we can observe convergence of velocity profile to a steady regime (Figure 5). The similar results we obtain when $\gamma=1$ and the function of fluid flow rate $Q(\tau)$ satisfies (Figure 6) and does not satisfy (Figure 7) the conditions of lemma. 


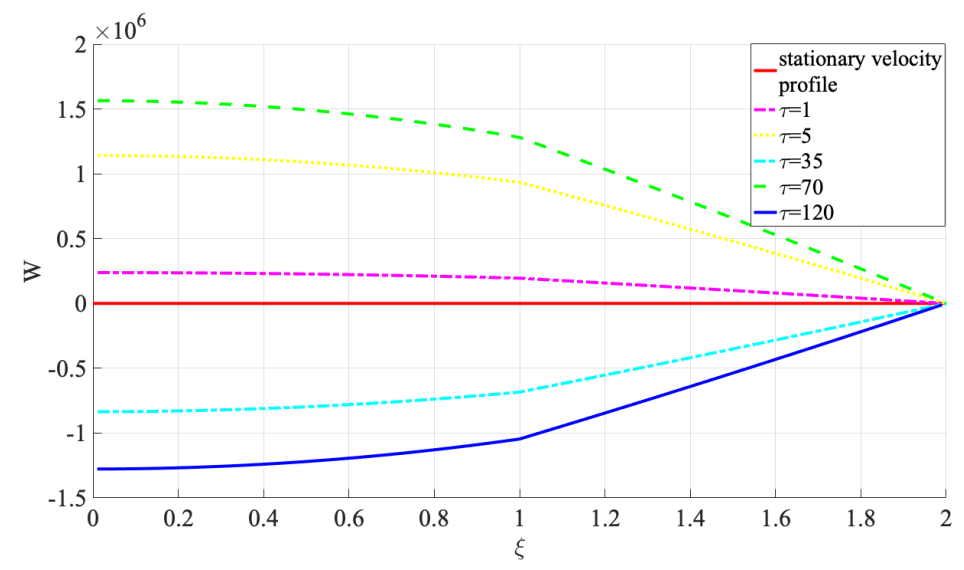

FIGURE 6. The dependence of the velocity profile on the radial coordinate at different values of the parameter $\tau$ when $\bar{Q}(\tau)=1+10000 \sin (0.1 \tau)$

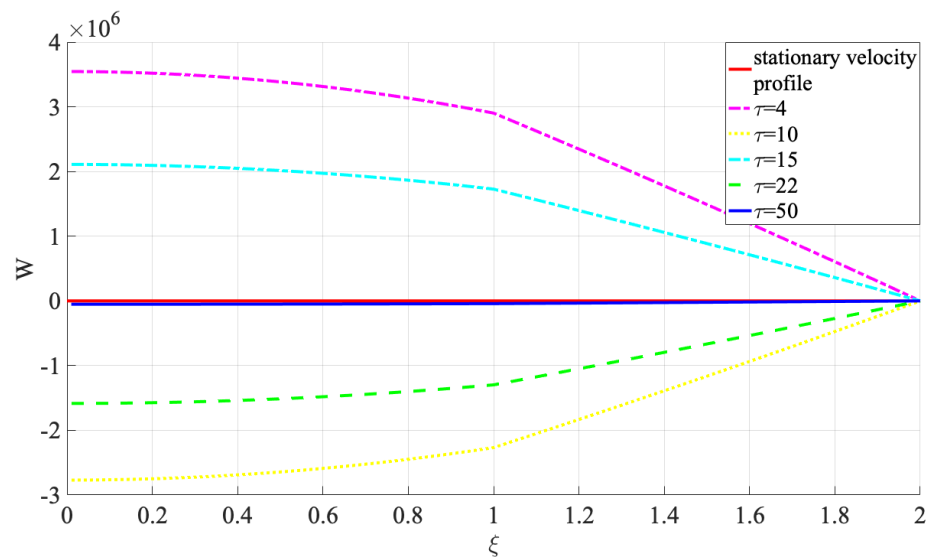

FIGURE 7. The dependence of the velocity profile on the radial coordinate at different values of the parameter $\tau$ when $\bar{Q}(\tau)=1-20000 \exp (-0.05 \tau) \sin (0.5 \tau)$

\section{CONCLUSION}

Thus the inverse problem about the two immiscible fluids motion with a cylindrical interface is investigated. The following results are obtained:

1. The stationary solutions for two types of volume flow specification are found.

2. In Laplace images, the solutions to non-stationary problems are obtained in quadratures, which contains Bessel functions.

3. It is shown that if the temperature on the solid tube wall uniformly tends to a stationary value with increasing time, then the solutions of the non-stationary problem with increasing time tend to a steady state.

4. On base of the Laplace transformation the numerical inversion the calculations results of the velocity and temperatures evolution are given. It has been established that due to the change in the temperature gradient on the tube wall, it is possible to control convective movement in the layer and cylinder.

\section{ACKNOWLEDGMENTS}

This research was supported by the Russian Foundation for Basic Research under grant 17-01-00229. 


\section{REFERENCES}

1. R. Kh. Zeytovnian, Convection in Fluids (Springer, Dordrecht - Heidelberg - London - New-York, 2009).

2. R.V. Birikh (1966) Thermocapillary convection in a horizontal layer of liquid, J. Appl. Mech. Tech. Phyz. 3, 69-72.

3. S. H. Davis, G. M. Homsy, and S. Rosenblat (1982) Nonlinear Marangoni convection in bounded layers. Part 1. Circular cylindrical containers, J. Fluid Mech. 120, 91-122.

4. P. C. Dauby, G. Lebob, and E. Bouhy (1997) Linear Benard-Marangoni instability in rigid circular containers, Phys. Rev. E56, 520-530.

5. H. C. Kuhlmann and U. Schoisswohl (2010) Flow instabilities in thermocapillary-buoyant liquid pools, J. Fluid Mech. 644, 509-535.

6. I. Hashim and S. K. Wilson (1999) The onset of Benard-Marangoni convection in a horizontal layer of fluid, Inter. J. of Engineering Science 37, 643-662.

7. V. K. Andreev, Yu. A. Gaponenko, O. N. Goncharova, and V. V. Pukhnachev, Mathematical Models of Convection (Walter de Gruyter, BerlinBoston, 2012).

8. M. A. Lavrentiev and B. V. Shabat, Methods of the Theory of Functions of a Complex Variable (Nauka, Moscow, 1973). [in Russian] 